\title{
Cerebellum Susceptibility to Neonatal Asphyxia: Possible Protective Effects of $\mathbf{N}$-Acetylcysteine Amide
}

\author{
T. Benterud $\mathbb{D}^{1,2,3}$ S. Manueldas, ${ }^{1}$ S. Rivera, ${ }^{4}$ E. Henckel, ${ }^{5,6}$ E. M. Løberg, ${ }^{3,7}$ S. Norgren, ${ }^{8}$ \\ L. O. Baumbusch, ${ }^{1}$ R. Solberg $\mathbb{D}^{1,},{ }^{1,2,9}$ and O. D. Saugstad ${ }^{1,3}$ \\ ${ }^{1}$ Department of Pediatric Research, Division of Pediatric and Adolescent Medicine, Oslo University Hospital, \\ Rikshospitalet, Oslo, Norway \\ ${ }^{2}$ Department for Surgical Research, Oslo University Hospital, Rikshospitalet, Oslo, Norway \\ ${ }^{3}$ University of Oslo, Oslo, Norway \\ ${ }^{4}$ Aix Marseille Université, CNRS, NICN, Marseille, France \\ ${ }^{5}$ Department of Clinical Science, Intervention and Technology, Karolinska Institutet, Stockholm, Sweden \\ ${ }^{6}$ Department of Neonatology, Karolinska University Hospital, Stockholm, Sweden \\ ${ }^{7}$ Department of Pathology, Oslo University Hospital, Ullevål, University of Oslo, Oslo, Norway \\ ${ }^{8}$ Department of Women's and Children's Health, Division of Paediatric Endocrinology, Karolinska Institutet, Stockholm, Sweden \\ ${ }^{9}$ Department of Pediatrics, Vestfold Hospital Trust, Tønsberg, Norway
}

Correspondence should be addressed to T. Benterud; torkil.benterud@rr-research.no

Received 1 May 2017; Revised 7 September 2017; Accepted 7 December 2017; Published 30 January 2018

Academic Editor: Hubertus Himmerich

Copyright (C) 2018 T. Benterud et al. This is an open access article distributed under the Creative Commons Attribution License, which permits unrestricted use, distribution, and reproduction in any medium, provided the original work is properly cited.

Background. After perinatal asphyxia, the cerebellum presents more damage than previously suggested. Objectives. To explore if the antioxidant N-acetylcysteine amide (NACA) could reduce cerebellar injury after hypoxia-reoxygenation in a neonatal pig model. Methods. Twenty-four newborn pigs in two intervention groups were exposed to $8 \%$ oxygen and hypercapnia, until base excess fell to $-20 \mathrm{mmol} / \mathrm{l}$ or the mean arterial blood pressure declined to $<20 \mathrm{mmHg}$. After hypoxia, they received either NACA (NACA group, $n=12$ ) or saline (vehicle-treated group, $n=12)$. One sham-operated group $(n=5)$ served as a control and was not subjected to hypoxia. Observation time after the end of hypoxia was 9.5 hours. Results. The intranuclear proteolytic activity in Purkinje cells of asphyxiated vehicle-treated pigs was significantly higher than that in sham controls $(p=0.03)$. Treatment with NACA was associated with a trend to decreased intranuclear proteolytic activity $(p=0.08)$, There were significantly less mutations in the mtDNA of the NACA group compared with the vehicle-treated group, $2.0 \times 10^{-4}\left( \pm 2.0 \times 10^{-4}\right)$ versus $4.8 \times 10^{-5}\left( \pm 3.6 \times 10^{-4}, p<0.05\right)$. Conclusion. We found a trend to lower proteolytic activity in the core of Purkinje cells and significantly reduced mutation rate of mtDNA in the NACA group, which may indicate a positive effect of NACA after neonatal hypoxia. Measuring the proteolytic activity in the nucleus of Purkinje cells could be used to assess the effect of different neuroprotective substances after perinatal asphyxia.

\section{Introduction}

Globally, approximately $45 \%$ of the cases of child death within the first five years of life occur during the neonatal period [1]. Despite the numbers of fatal cases due to the complications of perinatal asphyxia have been remarkably reduced over the last 15 years, there are still many children suffering from extensive neurological consequences after perinatal asphyxia.

It is widely recognized that in neonatal basal ganglia, the cerebral cortex, thalamus, and hippocampus are the most vulnerable brain areas after perinatal hypoxia [2]. However, improved neuroimaging modalities have shown that the cerebellum is more damaged after perinatal 
asphyxia than previously suggested [3]. In addition to being a coordinator of motor function, the cerebellum plays a role in higher cognitive functions and several authors argue that the abnormalities in the cerebellum may play a pivotal role in different mental disorders, such as attention deficit and hyperactivity disorder (ADHD) and schizophrenia $[4,5]$.

Our group has recently described anti-inflammatory and possible neuroprotective effects of the antioxidant $\mathrm{N}$-acetylcysteine amide (NACA) after neonatal hypoxiareoxygenation in a neonatal pig model. Further, NACA reduced the levels of the proinflammatory cytokine IL$1 \beta$ and the transcription factor NF- $\kappa \mathrm{B}$ in the prefrontal cortex of the brain after neonatal hypoxia-reoxygenation [6]. The substance NACA has many similarities with $\mathrm{N}$ acetylcysteine, which has been used as an antioxidant precursor to glutathione in the treatment of paracetamol overdose for more than 30 years [7]. However, due to the amide group which increases its lipophilicity, NACA has an augmented ability to penetrate the blood-brain barrier and the cellular membranes $[8,9]$. Moreover, we showed in a pig epithelial-like embryonic EFN-R kidney cell line that NACA had a protective effect on cells exposed to $\mathrm{H}_{2} \mathrm{O}_{2}$-induced oxidative stress [10].

In the present study, we wanted to explore if NACA treatment after hypoxia-reoxygenation reduces cerebellar injury, using the same group of pigs. The model is well established, and it has been used for years in our department to induce oxidative stress [11].

Matrix metalloproteinases (MMPs) display proinflammatory activity and exert deleterious actions in numerous neuropathological settings, including hypoxia and ischemia $[12,13]$. Moreover, some MMPs have been located in the nucleus of neural cells $[14,15]$ and are associated with neuronal DNA degradation upon oxygen-glucose deprivation [16]. Therefore, we used in situ zymography, which reflects the net metalloproteinase activity in the tissue, to measure proteolytic activity in the cerebellum upon hypoxia-reoxygenation.

Furthermore, reactive oxygen species (ROS) produced during and after perinatal asphyxia may induce lesions of mitochondrial DNA (mtDNA) and subsequently lead to impaired function of neural cells [17]. In this study, we investigated mtDNA in the cerebellum after hypoxiareoxygenation and if the pigs subjected to NACA after hypoxia (NACA group) would be less susceptible to mutations of mtDNA. The objective of the present study was to evaluate the damage-reduction potential of NACA on cerebellar injury after hypoxia-reoxygenation in neonatal pigs.

\section{Methods}

2.1. Study Design. A total of 29 newborn pigs, age 12-36 hours, hemoglobin $>5 \mathrm{~g} / \mathrm{dl}$, and in good general condition, were included in this study (Figure 1). The pigs were anesthetized, ventilated, and surgically prepared, including insertions of central venous and arterial lines, as previously described by Benterud et al. [18].
The experimental protocol has been thoroughly described in our previous article [6]. Briefly summarized, twenty-four pigs were randomized into two intervention groups. Both groups were subjected to $8 \%$ oxygen until base excess (BE) values declined to $-20 \mathrm{mmol} / \mathrm{l}$ or mean arterial blood pressure (MABP) fell below $20 \mathrm{mmHg}$. During hypoxia, $\mathrm{CO}_{2}$ was added, to achieve a $\mathrm{PaCO}_{2}$ of $8.0-9.5 \mathrm{kPa}$, in order to imitate perinatal asphyxia. At the end of hypoxia, 12 of the pigs were treated with NACA $300 \mathrm{mg} / \mathrm{kg}$ diluted in saline $0.9 \%$, while the other 12 received normal saline (vehicle-treated group). An additional dose of NACA or saline was administered 270 minutes after the hypoxic challenge. The pigs were reoxygenated with air for 9.5 hours until they were terminated with an overdose of pentobarbital $150 \mathrm{mg} / \mathrm{kg}$.

The five pigs in the sham-operated group underwent the same procedures as described in our previous article and were not exposed to hypoxia.

Due to the fact that there might be some subtle gender differences between neonatal pigs [19], the same number of male and female animals were included in each group.

\section{Laboratory Methods}

3.1. In Situ Zymography. We focused our investigations of the cerebellum on Purkinje cells, due to their high vulnerability to hypoxia [20, 21]. Furthermore, the nucleus of Purkinje cells presented the highest level of fluorescence and seemed to be particularly affected by hypoxia-reoxygenation. In situ zymography is commonly used as an index of net metalloproteinase activity resulting from the balance between gelatinases (principally MMP-9 and MMP-2) and the tissue inhibitors of MMPs (TIMPs) that are present in the tissue. In situ zymography was performed to localize net gelatinolytic activity in cerebellar brain sections, with minor modifications compared to the method previously described for brain tissue [22]. Sections of fresh frozen brain tissue ( $20 \mu \mathrm{m}$ thick) from the cerebellum were generated using a cryostat (Leica CM3050S, Nussloch, Germany). Nonfixed brain sections were incubated for 2 hours at $37^{\circ} \mathrm{C}$ in a humid dark chamber in a reaction buffer that contained $0.5 \mathrm{M}$ Tris- $\mathrm{HCl}, 1.5 \mathrm{M}$ $\mathrm{NaCl}, 50 \mathrm{mM} \mathrm{CaCl}, 2 \mathrm{mM}$ sodium azide ( $\mathrm{pH} 7.6$ ), and $80 \mu \mathrm{g} / \mathrm{ml}$ of intramolecularly quenched FITC-labeled DQgelatin (EnzCheck collagenase assay kit; Thermo Fisher Scientific, Waltham, Massachusetts, USA). After the incubation, the tissue was fixed in 4\% paraformaldehyde Antigenfix solution (Diapath, MM France, Brignais, France), incubated for 5 minutes with $0.5 \mu \mathrm{g} / \mathrm{ml}$ Hoechst 33258 (Thermo Fisher Scientific), and mounted in Prolong Gold Antifading reagent (Thermo Fisher Scientific). The sections were incubated with $1 \mathrm{mM}$ phenanthroline (Thermo Fisher Scientific), a broad-spectrum metalloproteinase inhibitor. Samples were observed with a confocal microscope (LSM 700 Zeiss, Jena, Germany), and images were analyzed using the Zen (Zeiss) and Image J softwares (NIH, Bethesda, MD, USA). GelatinFITC cleavage by tissue gelatinases releases quenched fluorescence representative of net proteolytic activity. Sections incubated without DQ-gelatin were not fluorescent. We used 8 piglets per experimental group and 5 from the control 


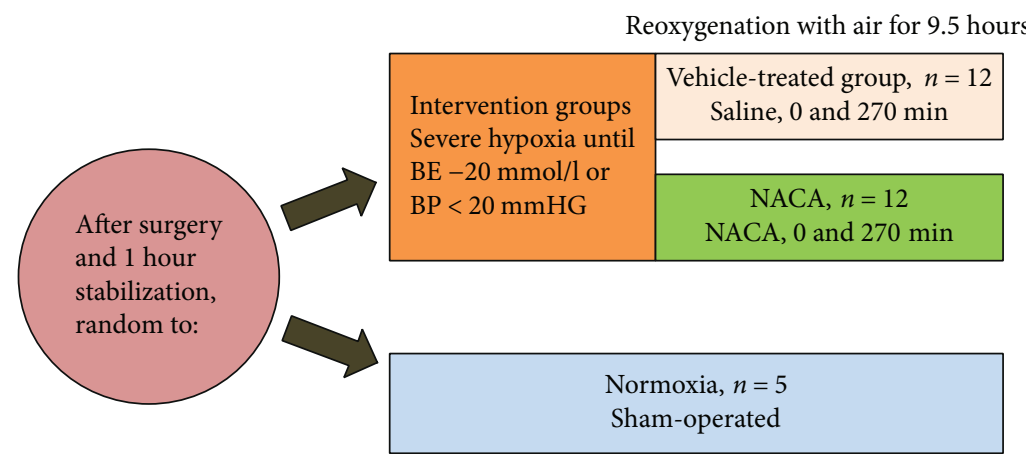

FIGURE 1: Experimental protocol: twenty-nine pigs were included, twelve in each intervention group and five in the sham group. $\mathrm{BE}=$ base excess and $\mathrm{BP}=$ blood pressure.

group, and we analyzed three slices per animal. The 8 pigs in each experimental group were randomly selected.

3.2. Histopathology. After removal of the brain, one hemisphere was immersion fixed in formalin. Tissue blocks $(0.5 \mathrm{~cm}$ thick $)$ from the cerebellum were embedded in paraffin, sliced in $4 \mu \mathrm{m}$ thick sections, and stained with hematoxylin and eosin (H\&E). An experienced neuropathologist evaluated the slices. Because of suboptimal conservation of the cerebellum, a simplified assessment was conducted. Cerebellar damage was categorized into two variables: (1) generalized damage and (2) localized/no damage.

The term generalized was used if the injuries were observed in all parts of the tissue section, in comparison to localized, where only small and limited parts of the tissue section were involved. Due to the limited amount of tissue, 23 pigs were evaluated, 5 in the sham group and 18 in the intervention groups. Of the pigs in the intervention groups, 8 were in the NACA group and 10 in the vehicle-treated group.

3.3. DNA Extraction from Cerebellum. Total DNA from the cerebellum was isolated using DNA blood and tissue kit (Qiagen, Hildesheim, Germany). $10-25 \mathrm{mg}$ of tissue from each pig was lysed and dissolved according to manufacturer's protocol with slight modifications (For a more detailed description, please read the Supplementary Materials (available here)).

3.4. Mutation Rate of Mitochondrial DNA. Random mutation capture (RMC) was performed to assess the rate of mutations of mtDNA in the cerebellum. The method is thoroughly described in the Supplementary Materials section.

3.5. Gene Expression. Real-time quantitative PCR (RT-qPCR) was performed to investigate the expression levels of genes involved in the NLRP3 inflammatory pathway, including IL-1 $\beta$, IL18, and NLRP3. RT-qPCRs were performed using the RT-RNA PCR kit following the instruction of the producer (Applied Biosystems, now Life 21 Technologies, Carlsbad, CA, USA). The final reaction volume was $25 \mu \mathrm{l}$, including $12.5 \mu \mathrm{l}$ of universal master mix (Life 21 Technologies), $200 \mathrm{nmol}$ of forward and reverse primers, and $5 \mu \mathrm{l}$ of the diluted cDNA product (1:12.5 dilutions). The 96-well plate reactions were carried out with an initial cycle at $50^{\circ} \mathrm{C}$ for 2 minutes, a heating stop at $95^{\circ} \mathrm{C}$ for 10 minutes, followed by 45 cycles of 30 seconds at $95^{\circ} \mathrm{C}$, and 60 seconds at $60^{\circ} \mathrm{C}$. All reactions were performed on a Vii7 Sequence Detection System (Life 21 Technologies). Experiments were performed in triplets, and all transcript quantification data were normalized to the endogenous reference gene $\mathrm{P} 0$.

The primer sequences $5^{\prime}-3^{\prime}$ were as follows: NLRP3 (forward primer) AAAAGCCTGAGTTGACCATTGTC and (reverse primer) CACTATCACTTATACACACCCAGATG TC; IL-1 $\beta$ (forward primer) GTGATGCCAACGTGCA GTCT and (reverse primer) GTGGGCCAGCCAGCACTA; and IL-18 (forward primer) GCCTCACTAGAGGTCTGGC AGTA and (reverse primer) GGACTCATTTCCTTAA AGGAAAGAGTT.

3.6. ELISA. To determine the protein concentrations of IL$1 \beta$, enzyme immunoassays kit was used as instructed by the manufacturer (R\&D Systems, Oxford, UK).

\section{Statistical Analysis}

The analyses were performed using SPSS software v21 (SPSS Inc., Chicago, IL, USA). The data were analyzed using the Kruskal-Wallis test, Mann-Whitney $U$ test, or Student $t$-test with winsorizing for variables with nonnormal distributions, ANOVA, and an independent sample $t$-test for normal distributions. Levene's test for equality of variance was performed before the $t$-test. If Levene's test documented a significant variance difference between the compared groups, a $t$-test assuming different variances was performed. Otherwise, a $t$-test assuming equal variance was performed.

All the differences were considered significant if $p<0.05$. When calculating the results of the histopathological evaluation, chi-square test without Yates' correction was performed.

\section{Results}

We did not find any significant difference between the genders, and therefore, the data for both genders are merged.

5.1. Physiological Parameters. At baseline, there were no differences in weight, hemoglobin, $\mathrm{pH}, \mathrm{BE}$, lactate, $\mathrm{pCO}_{2}$, or 
TABLE 1: Background and physiological parameters throughout the experiment.

\begin{tabular}{|c|c|c|c|}
\hline & Control $n=5$ & Hypoxia + NACA $n=12$ & Hypoxia + saline $n=12$ \\
\hline Weight (g) & $1923( \pm 76)$ & $1874( \pm 184)$ & $1924( \pm 124)$ \\
\hline Hypoxia time (min) & $33( \pm 12)$ & $40( \pm 13)$ & $33( \pm 12)$ \\
\hline $\mathrm{Hb} \mathrm{g} / 100 \mathrm{ml}$ start & $7.7( \pm 1.8)$ & $8.0( \pm 1.2)$ & $7.0( \pm 1.0)$ \\
\hline $\mathrm{Hb} \mathrm{g} / 100 \mathrm{ml}$ end & $5.9( \pm 1.2)$ & $6.3( \pm 0.8)$ & $7.1( \pm 2.7)$ \\
\hline Gender (male/female) & $3 / 2$ & $6 / 6$ & $6 / 6$ \\
\hline \multicolumn{4}{|l|}{$\mathrm{pH}$} \\
\hline Start & $7.44( \pm 0.04)$ & $7.42( \pm 0.04)$ & $7.45( \pm 0.04)$ \\
\hline End hypoxia & $7.43( \pm 0.05)$ & $6.87( \pm 0.08)$ & $6.92( \pm 0.11)$ \\
\hline 30 min reox & $7.46( \pm 0.04)$ & $7.18( \pm 0.09)$ & $7.18( \pm 0.07)$ \\
\hline $90 \min$ reox & $7.45( \pm 0.06)$ & $7.45( \pm 0.04)$ & $7.35( \pm 0.08)$ \\
\hline 270 min reox & $7.42( \pm 0.12)$ & $7.45( \pm 0.04)$ & $7.39( \pm 0.10)$ \\
\hline 570 min reox & $7.38( \pm 0.06)$ & $7.42( \pm 0.04)$ & $7.39( \pm 0.09)$ \\
\hline \multicolumn{4}{|l|}{$\mathrm{BE}(\mathrm{mmol} / \mathrm{l})$} \\
\hline Start & $4.3( \pm 2.9)$ & $-0.5( \pm 4.9)$ & $-0.3( \pm 3.6)$ \\
\hline End hypoxia & $4.1( \pm 3.6)$ & $-18.9( \pm 2.2)$ & $-19.0( \pm 3.9)$ \\
\hline 30 min reox & $4.4( \pm 1.9)$ & $-13.3( \pm 4.7)$ & $-15.1( \pm 3.5)$ \\
\hline $90 \min$ reox & $4.2( \pm 2.3)$ & $-3.9( \pm 5.0)$ & $-5.7( \pm 4.6)$ \\
\hline $270 \min$ reox & $2.9( \pm 4.5)$ & $-2.2( \pm 4.4)$ & $-1.4( \pm 5.0)$ \\
\hline 570 min reox & $0.5( \pm 3.4)$ & $-6.5( \pm 6.6)$ & $-3.7( \pm 5.4)$ \\
\hline \multicolumn{4}{|l|}{ Lactate $(\mathrm{mmol} / \mathrm{l})$} \\
\hline Start & $2.3( \pm 1.1)$ & $2.3( \pm 1.1)$ & $2.8( \pm 1.0)$ \\
\hline End hypoxia & $2.4( \pm 2.1)$ & $14.3( \pm 2.7)$ & $13.2( \pm 3.1)$ \\
\hline 30 min reox & $1.8( \pm 1.1)$ & $10.7( \pm 3.8)$ & $11.2( \pm 0.6)$ \\
\hline 90 min reox & $1.5( \pm 0.6)$ & $5.9( \pm 2.2)$ & $6.3( \pm 2.1)$ \\
\hline 270 min reox & $1.3( \pm 0.4)$ & $1.7( \pm 0.8)$ & $2.3( \pm 2.2)$ \\
\hline 570 min reox & $1.7( \pm 1.0)$ & $1.4( \pm 1.0)$ & $2.1( \pm 2.0)$ \\
\hline \multicolumn{4}{|l|}{ Glucose (mmol/l) } \\
\hline Start & $5.0( \pm 1.2)$ & $6.4( \pm 2.1)$ & $6.7( \pm 2.3)$ \\
\hline End hypoxia & $5.0( \pm 1.5)$ & $9.6( \pm 3.4)$ & $9.1( \pm 3.7)$ \\
\hline 30 min reox & $4.7( \pm 0.8)$ & $8.0( \pm 3.5)$ & $7.5( \pm 3.6)$ \\
\hline 90 min reox & $4.9( \pm 0.6)$ & $6.5( \pm 2.3)$ & $6.7( \pm 2.3)$ \\
\hline \multicolumn{4}{|l|}{$\mathrm{pCO}_{2}(\mathrm{kPa})$} \\
\hline Start & $5.0( \pm 1.1)$ & $5.2( \pm 0.9)$ & $5.0( \pm 1.1)$ \\
\hline End hypoxia & $5.7( \pm 0.3)$ & $8.4( \pm 1.4)$ & $7.7( \pm 1.1)$ \\
\hline $30 \min$ reox & $5.4( \pm 0.6)$ & $5.1( \pm 0.8)$ & $4.4( \pm 0.6)$ \\
\hline
\end{tabular}

glucose level between the groups. Arterial blood gases were taken at 6 different time points during the experiment. There were no significant differences between the 2 intervention groups in any of these variables. The physiological parameters and their change during the experiments are thoroughly described in Table 1.

5.2. In Situ Zymography. Compared to sham animals, a significant increased proteolytic activity was found in animals exposed to hypoxia alone (vehicle group, $p=0.03$ ), by contrast to NACA animals where a significant difference was not found $(p=0.08)$. Representative images obtained from five (sham) and eight (NACA and vehicle-treated groups) animals in each group are shown in Figure 2.
5.3. Histopathology. Significantly, more pigs in the intervention groups had generalized damage than in the control group $(p<0.05)$ (Table 2$)$.

There was no difference between the two intervention groups $(p=0.67)$.

Figure 3 shows an example of the cerebellum of a pig with a localized damage in different magnifications.

5.4. mtDNA Mutation. There were significantly fewer mutations in the NACA group than in the vehicle-treated group $\left(2.0 \times 10^{-4}, \mathrm{SD} \pm 2.0 \times 10^{-4}\right.$ versus $\left.4.9 \times 10^{-4}, \mathrm{SD} \pm 3.6 \times 10^{-4}\right)$ $(p<0.05)$ (Figure 4).

There was no significant difference when comparing the mtDNA mutation rate between the sham $\left(2.2 \times 10^{-4}\right.$, 

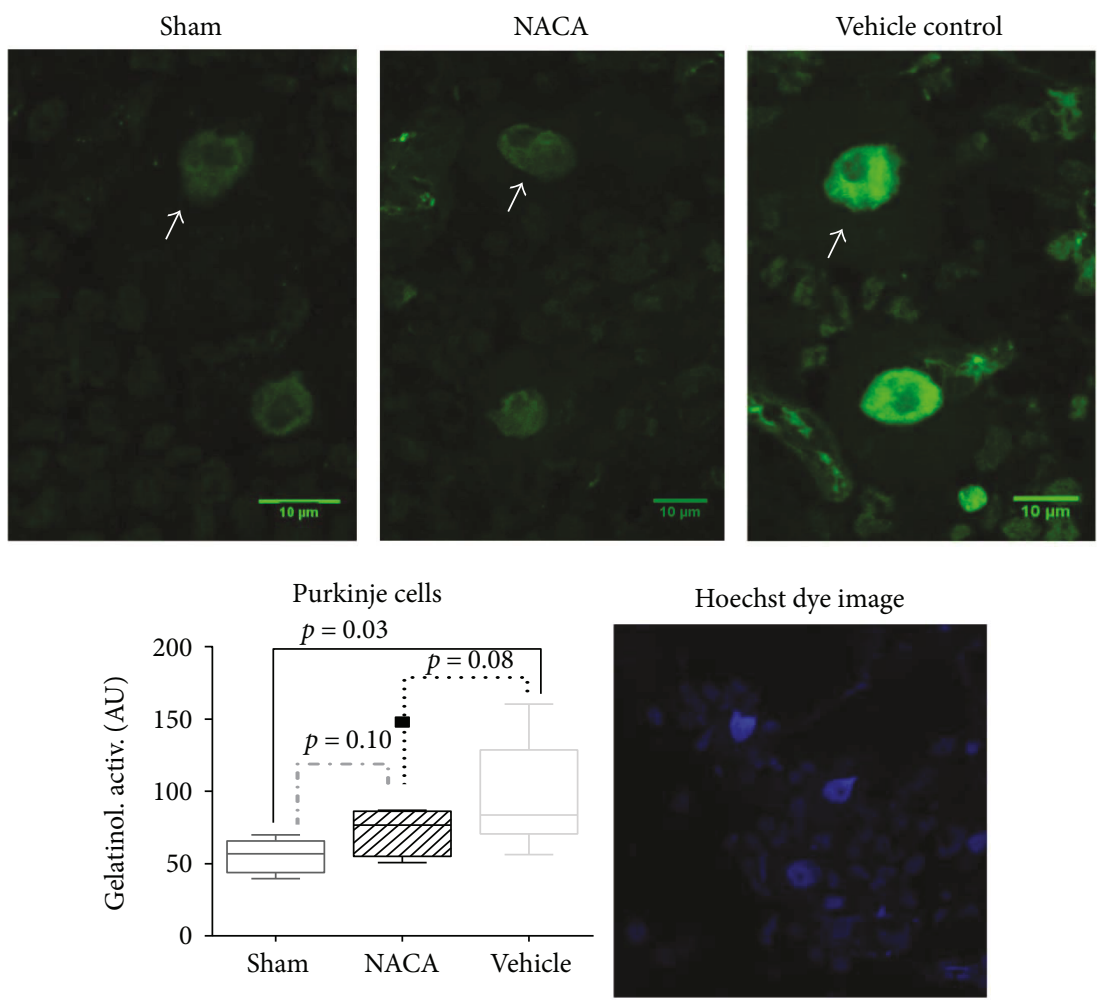

Figure 2: In situ zymography of the cerebellum. Net in situ gelatinolytic activity increases in the nucleus of Purkinje cells in the cerebellum after hypoxia-resuscitation. Fluorescence photomicrographs of cerebellar sections displaying in situ zymography in pigs who were shamoperated, exposed to NACA after hypoxia, and vehicle-treated groups. Intranuclear fluorescence signal in Purkinje cells (white arrow) represents the proteolytic activity (green). An increase in fluorescence signal strength represents a higher degree of proteolytic activity. The graph represents the quantification of net gelatinolytic activity (in arbitrary units (AU) of fluorescence) for sham 52 ( \pm 12 ), NACA group $72( \pm 16)$, and vehicle-treated group $97( \pm 36)$. Values are given as mean \pm SD. Hoechst dye was used as a nuclear marker (blue). Images are representative of pictures obtained from each group.

TABLE 2

\begin{tabular}{lccc}
\hline & $\begin{array}{c}\text { Generalized } \\
\text { damage }\end{array}$ & Localized or no damage & Total \\
\hline Intervention & 9 & 9 & 18 \\
Sham & 0 & 5 & 5 \\
Total & 9 & 14 & 23 \\
\hline
\end{tabular}

$\left.\mathrm{SD} \pm 1.7 \times 10^{-4}\right)$ and vehicle-treated groups $(p=0.11)$; however, the sham group consists of only five pigs.

5.5. Quantitative Real-Time PCR ( $q R T-P C R)$. Between the groups, there were no significant differences in gene expression of NLRP3, IL-18, and IL-1 $\beta$.

5.6. Protein Concentrations of $I L-1 \beta$ in the Cerebellum. The concentrations of IL-1 $\beta$ did not differ between the groups. In the NACA group, the concentration was $15.9 \pm 9.6$ versus $16.9 \pm 8.2$ in the vehicle-treated group $(p=0.82)$. A figure of the concentrations of IL- $1 \beta$ is included in the Supplementary Materials.

\section{Discussion}

To our knowledge, the present study is the first to use in situ zymography of the cores of Purkinje cells as a marker of hypoxic damage. Measuring the net gelatinolytic activity may be a relevant method to assess the grade of inflammation in cerebellar tissue. Net gelatinolytic activity reflects the proteolytic activity of gelatinases in a specific tissue [22]. The proteolytic activity of MMPs is tightly associated with the activity of TIMPs. Altered balance between MMPs and TIMPs results in a less-controlled equilibrium and may lead to an abrupt increase of proteolysis and pathological processes, including inflammation $[12,13]$. This assumption has been strengthened by numerous observations relating to increases in gelatinase activity with glial reactivity and neuronal demise and has been demonstrated in rodents after global cerebral ischemia $[22,23]$ and excitotoxic seizures induced by kainate [24]. In the latter model, gelatinolysis increased in neurons as early as eight hours after excitotoxic insult and remained high for several days in blood vessels and reactive glial cells of vulnerable areas, in relation with neuroinflammation.

Moreover, Chen et al. showed that exposing newborn rats to a broad-spectrum inhibitor of MMPs after hypoxiaischemia provided a long-term protection in both neuronal morphology and neurological function in the immature 


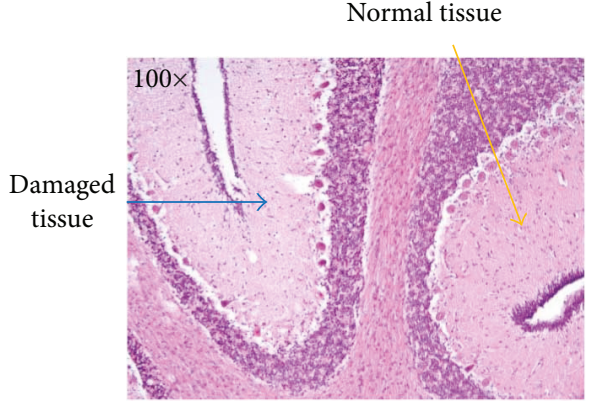

(a)

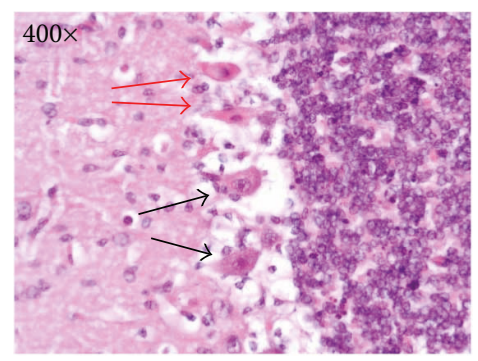

(b)

FIGURE 3: An example of localized damage in the cerebellum from one pig (two different magnifications 100x and 400x). In (b), the red arrows point to eosinophilic Purkinje cells, representing neurons with hypoxic injury. The black arrows point to normal Purkinje cells.

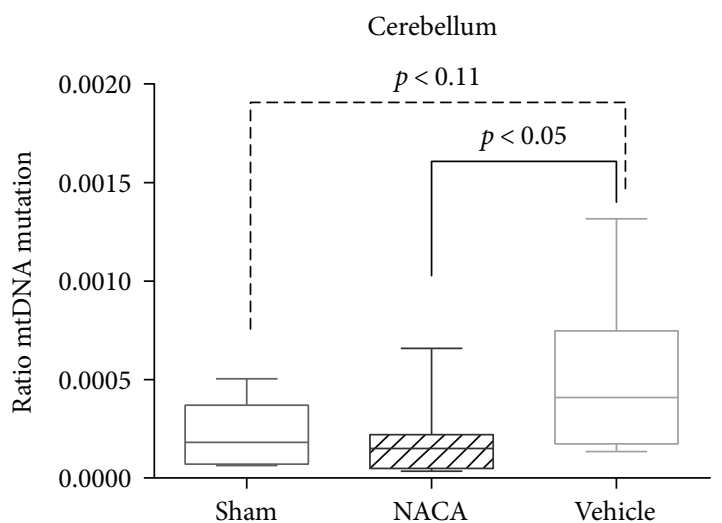

FIgURE 4: The picture depicts mtDNA mutations in the cerebellum. Values are given as mean $\pm \mathrm{SD}$. When comparing the ratios of mutations between the different groups, there was a significantly lower ratio of mutations in pigs exposed to NACA after severe hypoxia than in vehicle-treated group $\left(2.0 \times 10^{-4}, \mathrm{SD} \pm 2.0 \times 10^{-4}\right.$ versus $\left.4.9 \times 10^{-4}, \mathrm{SD} \pm 3.6 \times 10^{-4}\right), p<0.05$.

brain [25]. Furthermore, Zhang et al. recently showed that net gelatinolytic activity was significantly increased after inflicted traumatic brain injury in a rodent model [26]. Taking these findings into consideration, we suggest that measuring net gelatinolytic activity in the nucleus of Purkinje cells has a stronger association with damage of the neurons than measuring mRNA or protein levels for gelatinases, because gelatinolysis provides the balance between proteolysis and its inhibition. Further on, we did not perform subcellular biochemical fractionation, since that could have compromised other analyses, for instance IL-1 $\beta$.

Our group has previously investigated the association between net gelatinolytic activity and gene expression of MMP-2 and MMP-9 in various tissues, such as the liver, lungs, and striatum $[27,28]$. The gene expression of MMP2 and MMP-9, as well as the protein levels of active MMP2 and MMP-9, was associated with increased activity in the liver and lungs, whereas no such association was found in the striatum. Due to the important role of Purkinje cells in the developing brain [29], we speculate that the analysis of gelatinolytic activity in these cells could serve as an important tool in evaluating the various effects of neuroprotective substances after hypoxia in different models. The observation that pigs treated with NACA had a tendency to lower levels of gelatinolytic activity, compared with the vehicle-treated group may indicate that NACA reduces inflammation in the cerebellum.

Our results are in line with previous investigations of our group by Solberg et al. on the striatum of neonatal piglets, which observed an increased gelatinolytic activity in the nuclear compartment as well as in the cytoplasm of the neurons 9.5 hours after hypoxia. At that time point, the differences between the groups were not visible on HE stainings [30]. Furthermore, Hill detected an increased intranuclear gelatinolytic activity immediately after reoxygenation in a primary culture of cortical neurons after oxygen and glucose deprivation [16]. In the same study, they treated rats with an inhibitor of MMPs before they were subjected to occlusion of the middle cerebral artery. The rats exposed to the MMP inhibitor displayed significantly less apoptosis than the control group. These observations may indicate that the increased intranuclear gelatinolytic activity in neurons, such as Purkinje cells, could be an early marker of future neuronal degeneration.

Regarding the gene expression of NLRP3, IL- $1 \beta$, and IL18, there were no significant changes between the groups. These results could be in line with previous findings by our group exhibiting that for rats exposed to hypoxia and sham-operated rats, the mRNA expression of these components were similar in some cerebral subregions, including the cortex and the subventricular zone, at 24 hours after hypoxia [31]. Therefore, it is not surprising that no variability between the groups was revealed at one specific time point in our study. Further studies should be conducted on the time dependency of the compounds of the NLRP3 inflammasome pathway.

In addition, investigations did not reveal any significant changes between the groups in the levels of IL- $1 \beta$ as early as 9.5 hours after hypoxia, which stands in contrast with another report, showing that cerebellar IL- $1 \beta$ concentrations were significantly changed for all time points between 3 hours and 7 days in neonatal rats subjected to hypoxia [32]. The differences between these studies could be due to different methodology or simply reflect specific reactions to hypoxic injury across animal species. 
An increased production of ROS may cause mutations in the mtDNA leading to a critical effect on the activity of the mitochondrial electron transport chain, which subsequently may lead to mitochondrial dysfunction, apoptosis/necrosis, and diseases [33]. Wang et al. showed that damage to mtDNA may lead to diminished mitochondrial bioenergetics and hamper the maturation of neural stem cells [34]. We speculate that our results consisting of a significant reduced mutation rate of mtDNA in pigs subjected to NACA after hypoxia may be associated with a better neurological outcome, which stands in line with the findings of Patel et al. who demonstrated that NACA preserved mitochondrial bioenergetics and improved functional recovery after inflicted spinal trauma [35]. The lack of significant difference between the sham-operated and vehicle-treated groups could be due to the limited number of pigs included in the sham group $(n=5)$.

Our findings suggest that NACA could reduce the mitochondrial damage and thereby have a positive influence on the energy metabolism of neural cells.

Histopathological analyses of the cerebellum revealed no significant differences between the intervention groups but a significant difference between the sham and the intervention groups. The limited signs of cell death observed in some of the sham pigs may be due to possible harmful effects of anesthesia on the brains of newborns, as described in other publications [36-38]. Moreover, all pigs underwent surgical procedures which could be potentially harmful. On the other hand, some anesthetics may have neuroprotective features and a recent study by Liu et al. demonstrated that midazolam may protect against neuroapoptosis induced by physiological and oxidative stress [39]. The anesthetic regime was similar for each pig, and therefore, these effects should be consistent across all animals.

After severe perinatal hypoxia, a certain degree of cell death will occur, during and immediately after the hypoxic challenge. Between 6 and 24 hours later, a phase of secondary energy failure may evolve with declines in phosphocreatinine and ATP, impaired mitochondrial function, and further neuronal cell death. Many animals in our study have probably not reached the phase of secondary energy failure, and there are few signs of cell death visible on histological sections 9.5 hours after hypoxia. At this time point, however, the mechanistic measures of injury may differ between animals exposed to NACA and those who were vehicle treated. We suggest that if we had run the trials for an extended period of time, we would have seen a difference in histopathology between the two intervention groups.

\section{Conclusion}

Generally, pigs exposed to NACA after hypoxia revealed a tendency to reduced gelatinolytic activity in cerebellar Purkinje cells, measured with in situ zymography and a significant reduction of the mutation rate of mtDNA in the cerebellum. We therefore speculate that NACA may have neuroprotective capabilities after perinatal asphyxia. Our observations are in line with our previous study using the same group of animals, where we described possible anti- inflammatory effects of NACA in the cortex of neonatal pigs [6]. However, more studies are needed before NACA could be considered useful in a neonatal clinical setting.

Finally, our results indicate that using in situ zymography in the investigation of Purkinje cells could be a valuable biomarker to compare different neuroprotective substances in hypoxia-reoxygenation models.

\section{Limitations of the Study}

The mutations of mtDNA were only measured at one time point. Gel zymography of MMPs was not conducted; however, we assume that evaluating the net gelatinolytic activity reflects the actual proteolytic activity better than measuring the activity of one specific MMP. Although animal experiments have largely contributed to our understanding of human pathophysiology, we should be cautious when translating the results from animals to humans. We postulate that the differences between the groups could have been larger if the animals had been observed over a longer time period. Also, we are aware that the inclusion of both genders in a small study could possibly attenuate observable differences.

Other points of concern are that the number of animals in each group was relatively small and the study time was limited to 9.5 hours, so there was no long-term follow-up. Furthermore, electrophysiological surveillance of the pigs with EEG while anesthetized could have provided us with valuable information; however, this was not performed in this trial.

Prior to this investigation, the interindividual distribution of the proteolytic activity and rate of mutations in mitochondrial DNA following asphyxia were unknown. Thus, a proper power calculation to determine the adequate sample size could not be performed. In retrospect, the small sample size is troublesome and limits the statistical analysis.

\section{Ethical Approval}

The Norwegian Council for Animal Research approved the experimental protocol (approval number 4630). The animals were cared for and handled in accordance with the European Guidelines for the Use of Experimental Animals by researchers who have been certified by the Federation of European Laboratory Animals Science Association (FELASA).

\section{Conflicts of Interest}

The authors declare no conflicts of interest.

\section{Acknowledgments}

The NACA used was a kind gift of Dr. Glenn Goldstein, New York, NY, USA. The authors thank Ms. Eliane Charrat for her technical support and Professor Sandvik for the advice on statistical analysis. The authors are also grateful for the assistance from Vivi Stubberud, Aurora Pamplona, Sera Sebastian, Leonid Pankratov, Bjørn Petter Benterud, Grethe 
Dyrhaug, Monica Atneosen-Åsegg, and Ashley Kim. The study was funded by Helse-Sør Øst (South and Eastern Norway Regional Health Authority; source number 6051; Project no. 39570).

\section{Supplementary Materials}

Cerebellum susceptibility to neonatal asphyxia: possible protective effects of $\mathrm{N}$-acetylcysteine amide (NACA). (Supplementary Materials)

\section{References}

[1] L. Liu, S. Oza, D. Hogan et al., "Global, regional, and national causes of child mortality in 2000-13, with projections to inform post-2015 priorities: an updated systematic analysis," The Lancet, vol. 385, no. 9966, pp. 430-440, 2015.

[2] J. D. Aridas, T. Yawno, A. E. Sutherland et al., "Detecting brain injury in neonatal hypoxic ischemic encephalopathy: closing the gap between experimental and clinical research," Experimental Neurology, vol. 261, pp. 281-290, 2014.

[3] S. Kwan, E. Boudes, G. Gilbert et al., "Injury to the cerebellum in term asphyxiated newborns treated with hypothermia," American Journal of Neuroradiology, vol. 36, no. 8, pp. 15421549, 2015.

[4] M. S. Salman and P. Tsai, "The role of the pediatric cerebellum in motor functions, cognition, and behavior: a clinical perspective," Neuroimaging Clinics of North America, vol. 26, no. 3, pp. 317-329, 2016.

[5] N. C. Andreasen and R. Pierson, "The role of the cerebellum in schizophrenia," Biological Psychiatry, vol. 64, no. 2, pp. 81-88, 2008.

[6] T. Benterud, M. B. Ystgaard, S. Manueldas et al., "N-Acetylcysteine amide exerts possible neuroprotective effects in newborn pigs after perinatal asphyxia," Neonatology, vol. 111, no. 1, pp. 12-21, 2017.

[7] O. Dean, F. Giorlando, and M. Berk, "N-Acetylcysteine in psychiatry: current therapeutic evidence and potential mechanisms of action," Journal of Psychiatry \& Neuroscience, vol. 36, no. 2, pp. 78-86, 2011.

[8] S. Penugonda and N. Ercal, "Comparative evaluation of Nacetylcysteine (NAC) and N-acetylcysteine amide (NACA) on glutamate and lead-induced toxicity in CD-1 mice," Toxicology Letters, vol. 201, no. 1, pp. 1-7, 2011.

[9] L. Grinberg, E. Fibach, J. Amer, and D. Atlas, "N-Acetylcysteine amide, a novel cell-permeating thiol, restores cellular glutathione and protects human red blood cells from oxidative stress," Free Radical Biology \& Medicine, vol. 38, no. 1, pp. 136-145, 2005.

[10] T. Benterud, S. Manueldas, S. Norgren, R. Solberg, O. D. Saugstad, and L. O. Baumbusch, "N-Acetylcysteine amide (NACA) reduces cell death after oxidative stress in a porcine embryonic kidney cell line," Journal of Biomedical Science and Engineering, vol. 10, no. 02, article 73809, 6 pages, 2017.

[11] H. C. Østerholt, I. Dannevig, M. H. Wyckoff et al., "Antioxidant protects against increases in low molecular weight hyaluronan and inflammation in asphyxiated newborn pigs resuscitated with $100 \%$ oxygen," PloS One, vol. 7, no. 6, article e38839, 2012.
[12] S. Rivera, M. Khrestchatisky, L. Kaczmarek, G. A. Rosenberg, and D. M. Jaworski, "Metzincin proteases and their inhibitors: foes or friends in nervous system physiology?," The Journal of Neuroscience, vol. 30, no. 46, pp. 15337-15357, 2010.

[13] K. Baranger, S. Rivera, F. D. Liechti et al., "Endogenous and synthetic MMP inhibitors in CNS physiopathology," Progress in Brain Research, vol. 214, pp. 313-351, 2014.

[14] O. Sbai, L. Ferhat, A. Bernard et al., "Vesicular trafficking and secretion of matrix metalloproteinases-2, -9 and tissue inhibitor of metalloproteinases-1 in neuronal cells," Molecular and Cellular Neurosciences, vol. 39, no. 4, pp. 549-568, 2008.

[15] O. Sbai, A. Ould-Yahoui, L. Ferhat et al., "Differential vesicular distribution and trafficking of MMP-2, MMP-9, and their inhibitors in astrocytes," Glia, vol. 58, no. 3, pp. 344-366, 2010.

[16] J. W. Hill, "Intranuclear matrix metalloproteinases promote DNA damage and apoptosis induced by oxygen-glucose deprivation in neurons," Neuroscience, vol. 220, pp. 277-290, 2012.

[17] A. H. Bhat, K. B. Dar, S. Anees et al., "Oxidative stress, mitochondrial dysfunction and neurodegenerative diseases; a mechanistic insight," Biomedicine \& Pharmacotherapy, vol. 74, pp. 101-110, 2015.

[18] T. Benterud, L. Pankratov, R. Solberg et al., "Perinatal asphyxia may influence the level of beta-amyloid (1-42) in cerebrospinal fluid: an experimental study on newborn pigs," PloS One, vol. 10, no. 10, article e0140966, 2015.

[19] A. Panzardi, M. L. Bernardi, A. P. Mellagi, T. Bierhals, F. P. Bortolozzo, and I. Wentz, "Newborn piglet traits associated with survival and growth performance until weaning," Preventive Veterinary Medicine, vol. 110, no. 2, pp. 206-213, 2013.

[20] J. Cervos-Navarro and N. H. Diemer, "Selective vulnerability in brain hypoxia," Critical Reviews in Neurobiology, vol. 6, no. 3, pp. 149-182, 1991.

[21] R. Hausmann, S. Seidl, and P. Betz, "Hypoxic changes in Purkinje cells of the human cerebellum," International Journal of Legal Medicine, vol. 121, no. 3, pp. 175-183, 2007.

[22] S. Rivera, C. Ogier, J. Jourquin et al., "Gelatinase B and TIMP-1 are regulated in a cell- and time-dependent manner in association with neuronal death and glial reactivity after global forebrain ischemia," European Journal of Neuroscience, vol. 15, no. 1, pp. 19-32, 2002.

[23] Z. Gu, M. Kaul, B. Yan et al., "S-Nitrosylation of matrix metalloproteinases: signaling pathway to neuronal cell death," Science, vol. 297, no. 5584, pp. 1186-1190, 2002.

[24] J. Jourquin, E. Tremblay, N. Decanis et al., "Neuronal activitydependent increase of net matrix metalloproteinase activity is associated with MMP-9 neurotoxicity after kainate," European Journal of Neuroscience, vol. 18, no. 6, pp. 1507-1517, 2003.

[25] W. Chen, R. Hartman, R. Ayer et al., "Matrix metalloproteinases inhibition provides neuroprotection against hypoxia-ischemia in the developing brain," Journal of $\mathrm{Neu}$ rochemistry, vol. 111, no. 3, pp. 726-736, 2009.

[26] S. Zhang, L. Kojic, M. Tsang et al., "Distinct roles for metalloproteinases during traumatic brain injury," Neurochemistry International, vol. 96, pp. 46-55, 2016.

[27] B. H. Munkeby, W. B. Borke, K. Bjornland et al., "Resuscitation of hypoxic piglets with $100 \% \mathrm{O}_{2}$ increases pulmonary metalloproteinases and IL-8," Pediatric Research, vol. 58, no. 3, pp. 542-548, 2005. 
[28] R. Solberg, J. H. Andresen, S. Pettersen et al., "Resuscitation of hypoxic newborn piglets with supplementary oxygen induces dose-dependent increase in matrix metalloproteinase-activity and down-regulates vital genes," Pediatric Research, vol. 67, no. 3, pp. 250-256, 2010.

[29] L. C. Hutton, E. Yan, T. Yawno, M. Castillo-Melendez, J. J. Hirst, and D. W. Walker, "Injury of the developing cerebellum: a brief review of the effects of endotoxin and asphyxial challenges in the late gestation sheep fetus," Cerebellum, vol. 13, no. 6, pp. 777-786, 2014.

[30] R. Solberg, E. M. Loberg, J. H. Andresen et al., "Resuscitation of newborn piglets. Short-term influence of $\mathrm{FiO}_{2}$ on matrix metalloproteinases, caspase-3 and BDNF," PloS One, vol. 5, no. 12, article e14261, 2010.

[31] M. B. Ystgaard, Y. Sejersted, E. M. Loberg, E. Lien, A. Yndestad, and O. D. Saugstad, "Early upregulation of NLRP3 in the brain of neonatal mice exposed to hypoxiaischemia: no early neuroprotective effects of NLRP3 deficiency," Neonatology, vol. 108, no. 3, pp. 211-219, 2015.

[32] C. Kaur, V. Sivakumar, Z. Zou, and E. A. Ling, "Microgliaderived proinflammatory cytokines tumor necrosis factoralpha and interleukin-1beta induce Purkinje neuronal apoptosis via their receptors in hypoxic neonatal rat brain," Brain Structure and Function, vol. 219, no. 1, pp. 151-170, 2014.

[33] H. A. Tuppen, E. L. Blakely, D. M. Turnbull, and R. W. Taylor, "Mitochondrial DNA mutations and human disease," Biochimica et Biophysica Acta (BBA) - Bioenergetics, vol. 1797, no. 2, pp. 113-128, 2010.

[34] W. Wang, P. Osenbroch, R. Skinnes, Y. Esbensen, M. Bjoras, and L. Eide, "Mitochondrial DNA integrity is essential for mitochondrial maturation during differentiation of neural stem cells," Stem Cells, vol. 28, no. 12, pp. 2195-2204, 2010.

[35] S. P. Patel, P. G. Sullivan, J. D. Pandya et al., "N-Acetylcysteine amide preserves mitochondrial bioenergetics and improves functional recovery following spinal trauma," Experimental Neurology, vol. 257, pp. 95-105, 2014.

[36] A. M. Brambrink, S. A. Back, A. Riddle et al., "Isofluraneinduced apoptosis of oligodendrocytes in the neonatal primate brain," Annals of Neurology, vol. 72, no. 4, pp. 525-535, 2012.

[37] A. J. Davidson, "Anesthesia and neurotoxicity to the developing brain: the clinical relevance," Paediatric Anaesthesia, vol. 21, no. 7, pp. 716-721, 2011.

[38] A. W. Loepke, "Developmental neurotoxicity of sedatives and anesthetics: a concern for neonatal and pediatric critical care medicine?," Pediatric Critical Care Medicine, vol. 11, no. 2, pp. 217-226, 2010.

[39] J. Y. Liu, F. Guo, H. L. Wu, Y. Wang, and J. S. Liu, "Midazolam anesthesia protects neuronal cells from oxidative stressinduced death via activation of the JNK-ERK pathway," Molecular Medicine Reports, vol. 15, no. 1, pp. 169-179, 2017. 


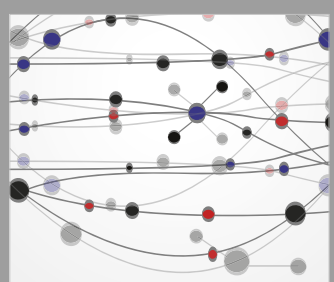

The Scientific World Journal
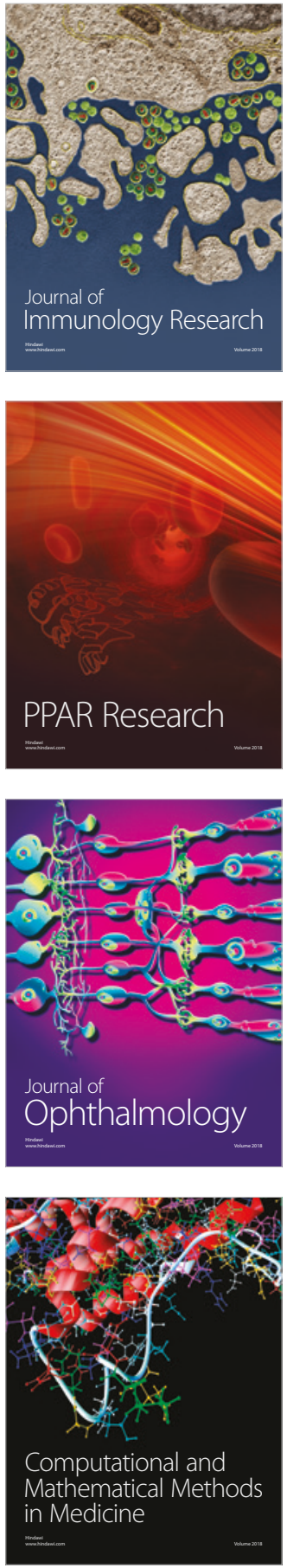

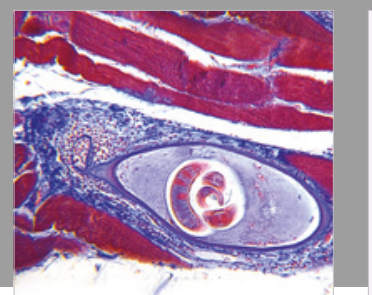

Gastroenterology Research and Practice

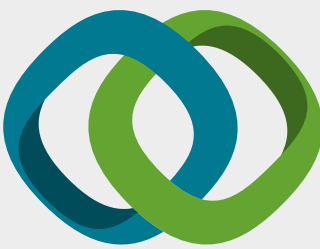

\section{Hindawi}

Submit your manuscripts at

www.hindawi.com
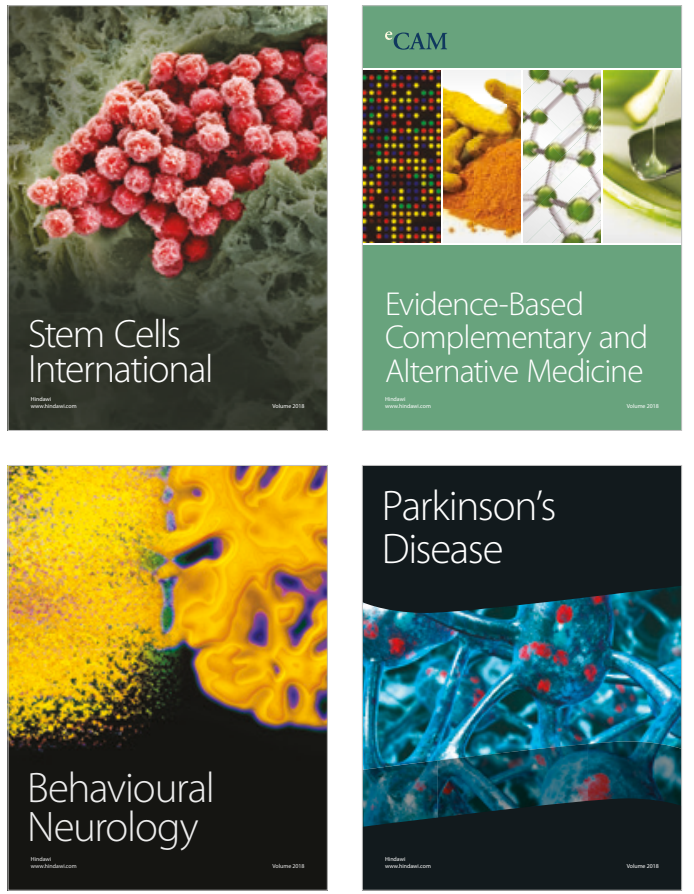

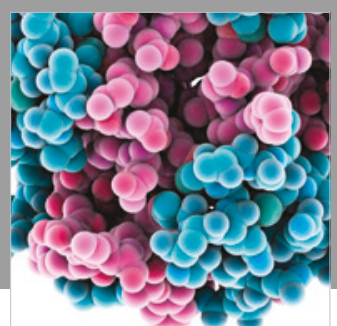

ournal of

Diabetes Research

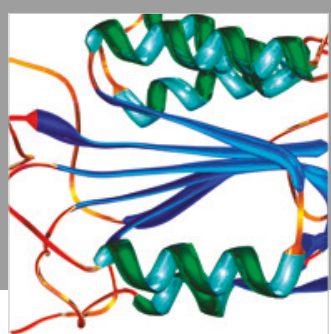

Disease Markers
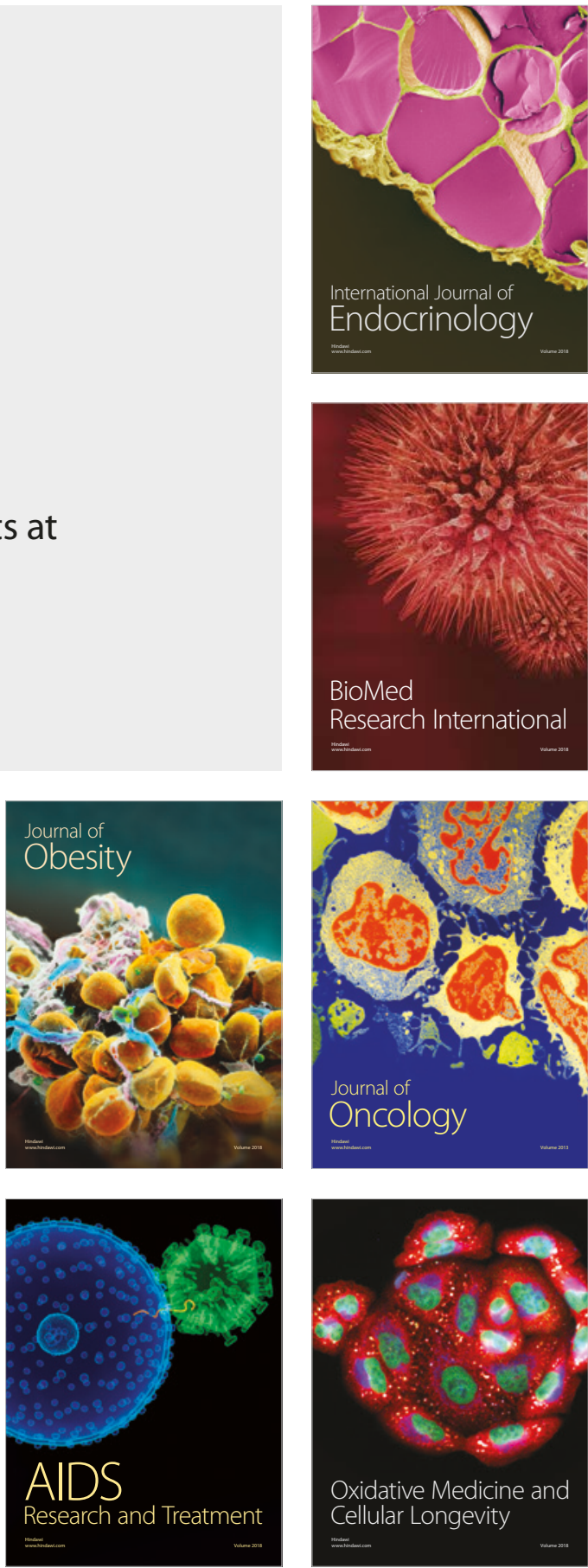